\title{
El papel de la genética en la aparición y desarrollo de la periodontitis. I: evidencias científicas de la asociación entre periodontitis y genética
}

\author{
RODRIGO-GÓMEZ D* \\ OTEO-CALATAYUD $\mathrm{A} * *$ \\ ALONSO-ROSADO $\mathrm{A} * * *$ \\ BASCONES-MARTINEZ A****
}

Rodrigo-Gómez D, Oteo-Calatayud A, Alonso-Rosado A, BasconesMartinez A. El papel de la genética en la aparición y desarrollo de la periodontitis. I: evidencias cientificas de la asociación entre periodontitis y genética. Av Periodon Implantol. 2007; 19, 2: 71-83.

\section{RESUMEN}

La periodontitis es una enfermedad multifactorial que se caracteriza por una reacción inflamatoria que afecta al aparato de inserción del diente. Esta inflamación es consecuencia de la interacción de ciertas bacterias con los mecanismos de respuesta inmune del huésped. Mientras que la infección es un requisito necesario para la aparición de la periodontitis, su curso y severidad depende de un número variable de determinantes ambientales, conductuales y genéticos. Se ha constatado que los individuos evolucionan de forma diferente ante el acúmulo de placa. Algunos, son muy susceptibles y desarrollan formas agresivas de periodontitis en edades relativamente tempranas y la pérdida de dientes se produce de forma generalizada en poco tiempo. Otros individuos nunca desarrollan periodontitis o la enfermedad progresa lentamente y la pérdida de dientes a lo largo de la vida del individuo es mínima.

Aunque en la patogénesis de la periodontitis la placa bacteriana es el factor etiológico primario, su presencia por si sola, no puede explicar la enorme variación de la enfermedad en la población. En este trabajo revisaremos el papel que la genética puede tener en los diferentes patrones de periodontitis analizando las bases científicas y clínicas que soportan esta cuestión.

\section{PALABRAS CLAVE}

Periodontitis, genética, polimorfismo, factor de riesgo.

Fecha de recepción: Diciembre 2006.

Fecha de aceptación: Enero 2007.

** Odontólogo por la U.E.M. Master en periodoncia e implantes por la U.C.M. Board of the European Federation of periodontology. Práctica privada en periodoncia e implantes en Segovia.

*** Dr. en Medicina y Cirugia USAL. Master en Periodoncia UCM. Profesor Asociado encargado de la asignatura de Periodoncia USAL. Practica Privada Peridoncia e Implantes en Salamanca

**** Catedrático de medicina bucal y periodoncia. Facultad de Odontología. U.C.M.. 


\section{INTRODUCCIÓN}

La periodontitis es una enfermedad multifactorial que resulta de la interacción de bacterias periodontopatógenas con los mecanismos de respuesta inmune del huésped y que se caracteriza por una reacción inflamatoria que afecta al aparato de inserción del diente. Las variaciones en el control inmunológico de la infección producida por las bacterias periodontopatógenas influencian la susceptibilildad del huésped a esta patología (1). Mientras que la infección es un requisito necesario para la aparición de la periodontitis, su curso y severidad depende de un número variable de determinantes ambientales, conductuales y genéticos (Esquema 1).

En muchos casos, de la literatura sobre enfermedades de carácter genético se engloba dentro de desórdenes en los que el factor genético es suficiente por si sólo para causar la enfermedad. Estas enfermedades, como la acondroplasia o la amelogénesis imperfecta suelen derivarse de la afectación de un solo gen y generalmente siguen patrones simples de transmisión mendeliana. Sin embargo, algunas enfermedades comunes como la osteoporosis o determinadas afectaciones coronarias tienen una base genética pero se comportan de forma diferente a los trastornos antes mencionados; Son enfermedades genéticas complejas o multifactoriales que habitualmente envuelven complejas interacciones de muchos genes y factores ambientales. En estas enfermedades multifactoriales, entre las que se encuentra la periodontitis, la genética no es, por si sola, suficiente para desencadenar la enfermedad con sus signos y síntomas y, al contrario que las de base estrictamente ge-

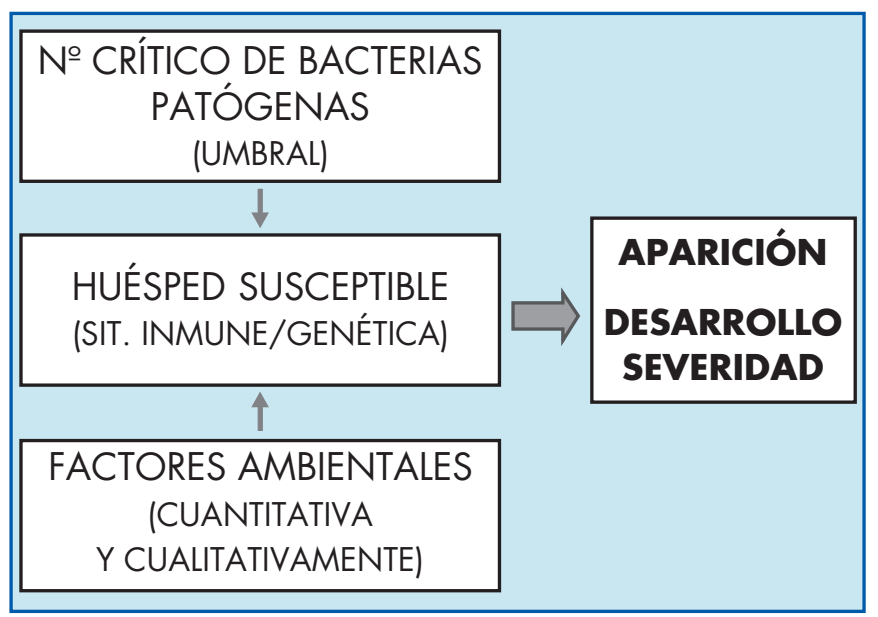

Esquema 1. nética, no suelen hacerse evidentes hasta la madurez (2). Las alteraciones genéticas en las enfermedades simples de transmisión mendeliana son normalmente mutaciones con importantes efectos biológicos y con capacidad por si solas de determinar el fenotipo de la enfermedad. El tipo de variaciones genéticas involucradas en las enfermedades genéticas complejas se denominan polimorfismos genéticos, son varios y normalmente sus efectos fenotípicos son más limitados.

La teoría de que el genotipo del huésped así como algunos factores ambientales influye en la patogenia de la enfermedad periodontal no es algo reciente. En 1930 Denny (62) concluyó tras revisar el estatus periodontal de varias familias que la susceptibilidad e inmunidad a la caries y la enfermedad periodontal eran probablemente heredables. Actualmente, se piensa que la actividad de la enfermedad periodontal depende de la presencia de un número crítico de patógenos en un huésped susceptible (3). El número y/o tipos de bacterias requeridos para superar el umbral individual de la enfermedad definen la susceptibilidad individual. Esta susceptibilidad puede estar influenciada por un gran número de determinantes genéticos y no genéticos. El propósito de este trabajo es revisar la emergente área en la periodoncia cuyo objetivo es medir y definir factores de riesgo genéticos del huésped para la enfermedad.

El trabajo se desarrollará en dos partes. En esta primera parte de la revisión nos vamos a centrar en los estudios científicos que evidencian una asociación entre periodontitis y genética. En una segunda parte, revisaremos esta asociación fundamentalmente sobre la base de las investigaciones de los polimorfismos más importantes en este campo.

\section{CONCEPTOS GENERALES}

El término "factor de riesgo" para la periodontitis hace referencia a un factor (biológico, medioambiental o conductual) confirmado por una secuencia temporal. Normalmente en estudios longitudinales, la presencia de un factor de riesgo incrementa de forma directa la probabilidad de que la enfermedad ocurra o suceda, y la ausencia del factor de riesgo reduce esta posibilidad (4). Existen factores de riesgo, como por ejemplo el tabaco, que son modificables. Por el contrario el genotipo es un factor no modificable.

Cuando hablamos de la composición genética de un organismo hablamos de su "genotipo", y la expresión 
del conjunto de sus rasgos o características se denomina fenotipo. El fenotipo esta determinado por la interacción de los genes y el ambiente. Los diferentes rasgos o enfermedades pueden estar causados por un simple gen (monogénicas), algunos genes (oligogénicas), o muchos genes (poligénicas). Las enfermedades cuya etiología incluye tanto factores genéticos como ambientales se denominan enfermedades multifactoriales. Las variaciones genéticas y diferentes exposiciones ambientales son la llave de los determinantes de las diferencias fenotípicas entre individuos.

Las localizaciones específicas de los genes en los cromosomas se denominan loci (locus en singular), y las variaciones en la secuencia de nucleótidos de un locus se denominan alelos. Para un locus dado, un individuo se considera "homocigoto" si los alelos son idénticos en los cromosomas homólogos y "heterocigoto" si los alelos son diferentes. Algunos alelos están asociados a cambios profundos en el fenotipo mientras otros no tienen prácticamente relevancia.

El término "marcador genético" se refiere a cualquier gen o secuencia de nucleótidos que pueda ser localizado en una zona o región específica de un cromosoma.

El patrón mediante el cual una enfermedad se transmite a través de las generaciones depende de cómo los alelos responsables de la enfermedad residen en cromosomas autosómicos o sexuales, de si son "recesivos" o "dominantes" y si son total o parcialmente penetrantes. De forma general un alelo dominante determina el fenotipo en un heterocigoto con otro alelo recesivo. Un alelo recesivo determina el fenotipo sólo si están presentes en ambos loci de un cromosoma homólogo. La penetrancia alude a la probabilidad de que un determinado fenotipo resulte de un genotipo, parcialmente penetrante significa que sólo una fracción de los individuos que heredan los alelos responsables de la enfermedad serán afectados.

Los genitistas se refieren a las diferentes formas de un gen como variantes alélicas o alelos. Cuando un alelo específico ocurre en al menos un $1 \%$ de la población se dice que es un "polimorfismo genético". Muchas variantes alélicas hacen referencia a cambios en la posición de uno de los 4 nucleótidos, algunas de estas variaciones alélicas alteran la composición de aminoácidos de las proteínas codificadas por genes. Cuando un cambio en un nucleótido es muy raro y no está presente en varios individuos se denomina mutación. Contrariamente a las mutaciones, los poli- morfismos genéticos son considerados variaciones normales de la población y pueden dar lugar, o no, a variaciones en el fenotipo.

Algunos polimorfismos que ocurren en genes no parecen cambiar el producto proteínico de los genes, sin embargo otros polimorfismos tienen un efecto relevante en la proteína codificada por el gen. Como contribuyen estos cambios al fenotipo de una enfermedad depende de las consecuencias específicas de la variación genética o del tipo de enfermedad. Ciertas exposiciones ambientales pueden ser determinantes importantes de cómo un alelo específico contribuye al riesgo de la enfermedad.

\section{EVIDENCIA DE LA SUSCEPTIBILIDAD GENÉTICA DE LA ENFERIMEDAD PERIODONTAL}

Desde hace muchos años se ha constatado que no todos los individuos evolucionan de la misma forma ante el acúmulo de placa. Algunos individuos son muy susceptibles y desarrollan formas agresivas de periodontitis en edades relativamente tempranas, mientras otros nunca desarrollan periodontitis. En algunos casos la enfermedad progresa lentamente y la pérdida de dientes a lo largo de la vida del individuo es mínima, mientras en otros su pérdida se produce de forma generalizada en poco tiempo.

Numerosos estudios han constatado la variabilidad en la susceptibilidad del huésped ante la agresión bacteriana. En investigaciones experimentales de gingivitis se ha podido comprobar la variación individual en el rango de aparición de inflamación gingival. Lindhe et al. (1978) (5) mostraron en un estudio experimental en perros, que el acumulo de placa a largo plazo y la aparición de gingivitis podían conducir a periodontitis. Sin embargo, mientras que en algunos perros se observaba pérdida de inserción, otros apenas la padecían. Wiedemann et al. (1979) (6) en otra investigación con un grupo de 62 pacientes a los que eliminaron cualquier procedimiento de higiene oral observaron que del grupo de estudio, ocho fueron "resistentes" y no desarrollaron gingivitis durante un periodo de observación de 21 días, otro grupo de 25 sujetos fue "susceptible" y exhibió inflamación gingival evidente en 14 días. Los demás sujetos constituyeron un grupo intermedio que desarrolló inflamación gingival en 21 días. En un estudio posterior, Van der Weijden et al. (1994)(7) describieron un grupo de pacientes con un grado de inflamación gingival que de forma consis- 
tente era superior a la media, mientras otro grupo permanecía con un nivel de inflamación por debajo de la misma. Las diferencias en la susceptibilidad a la gingivitis entre los dos grupos no pudieron achacarse a diferencias cualitativas en la placa.

Estas observaciones en gingivitis concuerdan con las obtenidas en periodontitis en los estudios longitudinales realizados en las plantaciones de te en Sri Lanmka (8). Estos estudios se realizaron con trabajadores en un ambiente muy parecido y con apenas higiene bucal o atención dental profesional (factores ambientales similares). Durante el tiempo de observación se les observaron tres patrones diferentes de periodontitis (1). Individuos que apenas desarrollaron enfermedad (11\%) (2), individuos que desarrollaron un patrón medio de la enfermedad (80\%) (3). Individuos con una progresión rápida y severa de la enfermedad (8\%). Estas variaciones se atribuyeron a un fuerte componente genético que marcaba diferentes susceptibilidades ante factores ambientales similares.

Como consecuencia de los resultados de todos estos trabajos, algunos conceptos tradicionales acerca del papel de la placa en la enfermedad periodontal cambiaron. Un cambio importante en la comprensión de la EP fue la observación de diferentes severidades en su prevalencia. En la década de los 80 se hizo evidente que la mayoría de los adultos sólo padecían gingivitis y que en aproximadamente un $30 \%$ se detectaban una o más localizaciones con periodontitis. De hecho, los casos con periodontitis generalizada severa la componen sólo un 8-13\% de la población adulta mundial $(8,9)$.

Partiendo del hecho de que la placa bacteriana es necesaria (aunque insuficiente) para la iniciación y progresión de la periodontitis, pacientes con un excelente control de placa apenas tendrán riesgo de padecer periodontitis, y pacientes con un pobre control de placa desarrollaran con mayor probabilidad la enfermedad. Sin embargo la mayoría de los pacientes están de alguna manera entre estos dos polos (higiene muy deficiente o excelente). De hecho, muchos de los pacientes que visitan a su dentista regularmente realizan su cepillado dental de una forma aceptable pero, o bien no utilizan dispositivos de limpieza interproximal o si lo hacen, no es de una forma correcta. Se ha postulado que es para este grupo de pacientes adultos, con una higiene oral entre moderada y buena en los que otros factores como el tabaco $(10,11)$, diabetes $(12,13) \circ$ algunos determinantes genéticos parecen influenciar de una forma más perceptible la aparición, desarrollo y severidad de la enfermad periodontal.
Aunque son destacables los progresos para tratar de clarificar la contribución de determinados factores genéticos en la etiología de la periodontitis, estos estudios muchas veces no diferencian los diversos papeles específicos que estos factores deben jugar en la patogénesis. Por ejemplo factores determinantes de la severidad de la periodontitis como el tabaco (10) son capaces de enmascarar la importancia de factores genéticos modificadores de la enfermedad que pueden ser importantes en ausencia del mismo $(14,15)$.

\section{Los estudios relacionados con el papel de los facto- res genéticos en la periodontitis utilizan con frecuen- cia poblaciones o grupos específicos de pacientes con determinadas formas de periodontitis. Sin em- bargo, la nomenclatura en el diagnóstico de los di- ferentes tipos de periodontitis ha cambiado. En este artículo de revisión utilizaremos el diagnóstico de periodontitis utilizado en los artículos originales. Esto incluye periodontitis de comienzo temprano (actualmente periodontitis agresiva) (16) subdividi- da en periodontitis prepuberal, juvenil y rápidamen- te progresiva. El término periodontitis del adulto se refiere actualmente a la periodontitis crónica (16).}

La evaluación de la significación de los estudios que analizan las implicaciones genéticas en la aparición y desarrollo de la periodontitis requieren de la comprensión de los métodos genéticos de análisis empleados. Existen varias técnicas para demostrar la base genética de las enfermedades. Algunos métodos son generales, mientras otros facilitan la identificación precisa de variaciones genéticas que causan o contribuyen a la enfermedad. Los métodos de análisis aquí expuestos han sido los más relevantes en la evaluación de los aspectos genéticos de la periodontitis.

\section{Estudios de agregación familiar}

La mayor parte de estos estudios se han centrado en periodontitis de comienzo temprano ahora denominadas periodontitis agresiva $(17,18,19,20)$. Hay que tener en cuenta que los patrones familiares pueden reflejar la exposición a factores ambientales comunes de dichas familias. Por tanto es importante considerar los factores de riesgo conductuales y ambientales compartidos en cualquier subgrupo familiar. Esto incluye educación, higiene oral, agrupación socioeconómica, enfermedades como la diabetes, fumadores pasivos, atención sanitaria, etc. 
Los intentos para correlacionar una respuesta celular, funcional e inmune con el fenotipo característico de la periodontitis de comienzo temprano en familias han sido muchas veces improductivos, excepto para indicar de forma evidente que un modelo simple de transmisión no es posible y que la periodontitis de comienzo temprano debe tener una etiología compleja y heterogénea $(21,22)$. Aunque se ha propuesto la transmisión de bacterias entre sujetos como una posible explicación de por qué la periodontitis de comienzo temprano se agrupa en determinadas familias, la observación de la transmisión de bacterias en familias es insuficiente por si sólo para explicar dicha agrupación familiar (22). La caracterización de los componentes etiológicos por tanto requiere de más estudios de análisis genéticos.

\section{Estudios de asociación}

Los estudios de asociación son utilizados para mostrar alelos responsables de enfermedad en regiones específicas de los cromosomas. Estos estudios, sirven para determinar la localización genética de un gen con un efecto fundamental sobre un determinado rasgo. En estas investigaciones se usan familias fijas o establecidas con muchos individuos afectados. El genotipo es determinado por los miembros de la familia afectados y no afectados, y se utilizan complejos modelos estadísticos para determinar como los alelos en cuestión y la enfermedad, se transmite en las familias bajo un modelo hereditario. Los parámetros que deben ser especificados incluyen el modelo de herencia, frecuencia del alelo en la población y la penetrancia de la enfermedad.

Para poner a prueba la asociación, se comparan la frecuencia de los alelos en un locus dado entre sujetos con enfermedad (casos) y sanos (controles) cogidos de la misma población. Los datos extraídos de este tipo estudios (casos-controles) han de ser interpretados con precaución ya que su diseño retrospectivo no permite establecer una temporalidad en la sucesión de los eventos y establecer una relación causa-efecto. Por tanto una asociación no implica necesariamente una conexión biológica entre el alelo estudiado y la enfermedad (23). Los estudios de asociación más destacados son los realizados por Boughman et al. 1986 (24) en Maryland y por Hart et al. 1993 (25) en 14 familias afroamericanas y 4 caucasianas. Estos estudios trataron de asociar la periodontitis agresiva con una región específica del cromosoma 4. En el primer estudio se encontró una asociación estadísticamente significativa que, sin embargo, no pudo ser corroborada por los trabajos de Hart y colaboradores. Los resultados dispares apoyan la heterogenicidad genética de la periodontitis agresiva.

\section{Estudios en gemelos}

El estudio de gemelos es uno de los modelos más interesantes para analizar la herencia de enfermedades. Los datos de gemelos son usados para estimar la hereditabilidad o proporción de una variación en el fenotipo atribuida a una variación genética. Una hereditabilidad del $50 \%$ significa que la mitad de la varianza en la población es atribuida a la variación genética. Para estimar la hereditabilidad con precisión son necesarias grandes muestras de gemelos criados juntos. También pueden obtenerse datos importantes del estudio de gemelos separados tras el nacimiento, ya que de esta forma se elimina el efecto de los factores ambientales y las similitudes entre estos gemelos pueden atribuirse de forma más específica al efecto de los genes compartidos.

Los estudios de gemelos más importantes son los conducidos en Minnesota por Michalowicz (26-29). Este grupo estudió los parámetros periodontales de una muestra amplia de gemelos monocigotos y dicigotos criados juntos y separados. Encontraron que entre un $38 \%$ y un $82 \%$ de la varianza de gingivitis y de los parámetros periodontales de profundidad de sondaje y pérdida de inserción de la población estudiada podían ser atribuidos a variaciones genéticas. Los gemelos monocigotos tuvieron una similitud mayor en las variables estudiadas que los gemelos dicigotos. Se encontró una variación genética estadísticamente significativa tanto para la severidad como para la extensión de la enfermedad. Se estimó una hereditabilidad del $50 \%$ para la periodontitis del adulto, que permaneció inalterada tras ajustar variables ambientales incluido el tabaco. Estos estudios concluyeron que aproximadamente la mitad de la variación de la periodontitis en la población puede ser atribuida a variaciones genéticas (29).

Los estudios de gemelos también se han usado para comprobar si los genes del huésped influyen en la composición de la microbiota oral. Así, se ha podido observar que los gemelos adolescentes tienen una microbiota más parecida que la de parejas de individuos no relacionados. (30). En los adultos sin embargo estas diferencias no son tan evidentes (29). 


\section{Análisis de segregación}

Los estudios de agregación son consistentes con el componente heredable de la periodontitis agresiva. Los de gemelos avalan el componente genético de la periodontitis del adulto. En los análisis de segregación, el patrón de enfermedad observado en las familias es comparado con los patrones esperados bajo varios modelos hereditarios. El poder estadístico de estos diseños depende del número y composición de las familias y de la heterogenicidad de las mismas. La heterogenicidad hace referencia a la presencia de diferentes causas de enfermedad entre las familias estudiadas. De forma general, los análisis de segregación tienen poco poder para resolver la heterogenicidad. Los análisis de segregación tampoco pueden distinguir entre los efectos propiamente genéticos y causas ambientales de la enfermedad, como la transmisión de patógenos dentro de las familias (31).

Los estudios más importantes de segregación realizados hasta el momento son los llevados a cabo por el grupo dirigido por Marazita et al (19), que estudiaron más de 100 familias y encontraron evidencias de una transmisión autosómica-dominante para la periodontitis agresiva. Concluyeron que esta hereditabilidad autosómica dominante tenía un $70 \%$ de penetrancia y afectaba de igual forma a negros y blancos. Actualmente la teoría más aceptada sobre la base genética de la periodontitis agresiva es que tanto la periodontitis prepuberal como la agresiva localizada y generalizada se deban probablemente al locus de un gen que es transmitido de forma autosómica dominante en Norte América con una penetrancia reducida y de forma autosómica recesiva en algunas poblaciones europeas (32). Estos modelos diferentes de transmisión reflejan la heterogenicidad genética de la enfermedad. La expresión penetrancia reducida hace referencia a que algunos sujetos con un determinado genotipo pueden no expresar el fenotipo. Esto es debido a que factores ambientales como el tabaco o el control de placa, pueden jugar un papel importante para permitir al fenotipo que se presente clínicamente (33).

\section{Periodontitis del adulto}

La periodontitis del adulto es la variante más frecuente de periodontitis, existiendo una serie de factores que van a desencadenar la aparición de la enfermedad. Como ya sabemos, la presencia de bacterias es fundamental para que se desarrolle la enfermedad. Sin la presencia de éstas no hay patología, sin embargo, se hacen necesarios la existencia de otros factores que favorezcan el inicio y progresión, como la respuesta inmunológica del paciente, el tabaco o algunas enfermedades sistémicas. Hay pacientes que a pesar de tener un control de placa aceptable y no fumar presentan una periodontitis del adulto más grave que pacientes con un control de placa peor y que fuman. Esta circunstancia plantea el papel subyacente que la susceptibilidad genética tiene en esta forma de periodontitis. De hecho algunos trabajos, como hemos revisado, (29) han observado que aproximadamente la mitad de la variabilidad clínica de la periodontitis crónica puede atribuirse a factores genéticos.

Uno de los primeros trabajos que demostraron la asociación de un determinado gen con la severidad de la periodontitis del adulto fue el realizado por Kornman et al (34). El trabajo se realizó con pacientes caucasianos no fumadores y se observó una asociación específica entre la severidad de la periodontitis y la presencia de un genotipo específico de la interleuquina-1 (IL-1). Funcionalmente, este genotipo específico comprende una variante en el gen de la IL-1B asociado con una elevada producción de IL-1. Estos resultados coinciden con los obtenidos en otros estudios con poblaciones geográficamente similares (35) Estudios posteriores (36) han investigado la prevalencia de la enfermedad en un grupo de pacientes categorizados en 9 regiones geográficas. Observaron que el $41 \%$ de los pacientes en el grupo de periodontitis moderada-severa eran IL-1 positivos frente al $28 \%$ del grupo periodontitis leve o no periodontitis. Además se constató una asociación significativa entre el consumo de tabaco y el genotipo de la IL-1 con la severidad de la periodontitis en función de la población de estudio desde un punto de vista étnico. Al analizar los resultados sólo en los pacientes de origen norte-europeo ( $82 \%$ de la muestra), se encontró un riesgo de sufrir periodontitis moderada-severa de $5.27 \mathrm{si}$ el paciente era IL-1+, si además estos pacientes eran fumadores, el riesgo se incrementaba en 1.09. Estos resultados revelan la importancia del análisis de los datos en función de un mismo origen geográfico/étnico. También muestran la fuerte asociación entre la susceptibilidad genética y un factor ambiental como el tabaco en la severidad de la periodontitis crónica del adulto.

\section{Periodontitis de comienzo temprano}

La mayor parte de las investigaciones sobre los factores genéticos en periodontitis se han centrado en las 
periodontitis de comienzo temprano. Actualmente estas formas están englobadas en la última clasificación sobre la enfermedad periodontal (16) como periodontitis agresiva. El interés se basa en la hipótesis de que a diferencia de la periodontitis del adulto, las formas más agresivas de periodontitis en pacientes jóvenes se deben en menor grado a la acción, con el transcurso del tiempo, de factores ambientales como la placa bacteriana o el tabaco. Además los pacientes con periodontitis de comienzo temprano representan probablemente una población más homogénea que los pacientes con periodontitis del adulto.

Dentro de la periodontitis de comienzo temprano y siguiendo la clasificación de la Academia Americana de Periodoncia de 1989 (37), que es la utilizada por la mayoría de estudios consultados en esta revisión, se incluyen una serie de subcategorías en base a las cuales vamos a desarrollar este apartado:

- Periodontitis prepuberal: Dentro de esta categoría, la respuesta inmune ante la agresión bacteriana, fundamentalmente el componente fagocítico, cobra un papel especialmente importante. En la periodontitis prepuberal generalizada se han constatado entre otras deficiencias leucocitarias, una disminución en la capacidad de adhesión de los leucocitos (38) que cursan con un reclutamiento anormal de neutrófilos y una respuesta inmune inadecuada. Para este tipo de periodontitis se ha reportado un patrón hereditario tanto autonómico recesivo (39) como dominante (40) (Figuras 1 A y B).

- Periodontitis juvenil: La prevalencia de esta enfermedad es de aproximadamente $2,49 \%$ en afroamericanos y $0^{\prime} 16 \%$ en caucasianos americanos. A pesar de ser una forma de enfermedad rara, se han descritos varios casos de enfermedad en una misma familia.

Históricamente se consideraba un proceso degenerativo no inflamatorio, aunque en la actualidad está clara la influencia de la placa en la etiología tanto de la periodontitis juvenil localizada (PJL) como de la generalizada (PJG). Aunque los primeros estudios sugirieron que la periodontitis juvenil se transmitía ligada al cromosoma $\mathrm{X}$ de forma dominante debido a su mayor prevalencia en mujeres y a su patrón de transmisión vertical, estudios recientes han revelado que probablemente esta forma de periodontitis se herede de forma autosómica recesiva $(32,41)$ o autosómica dominante (19) quizás esta inconsistencia en los datos
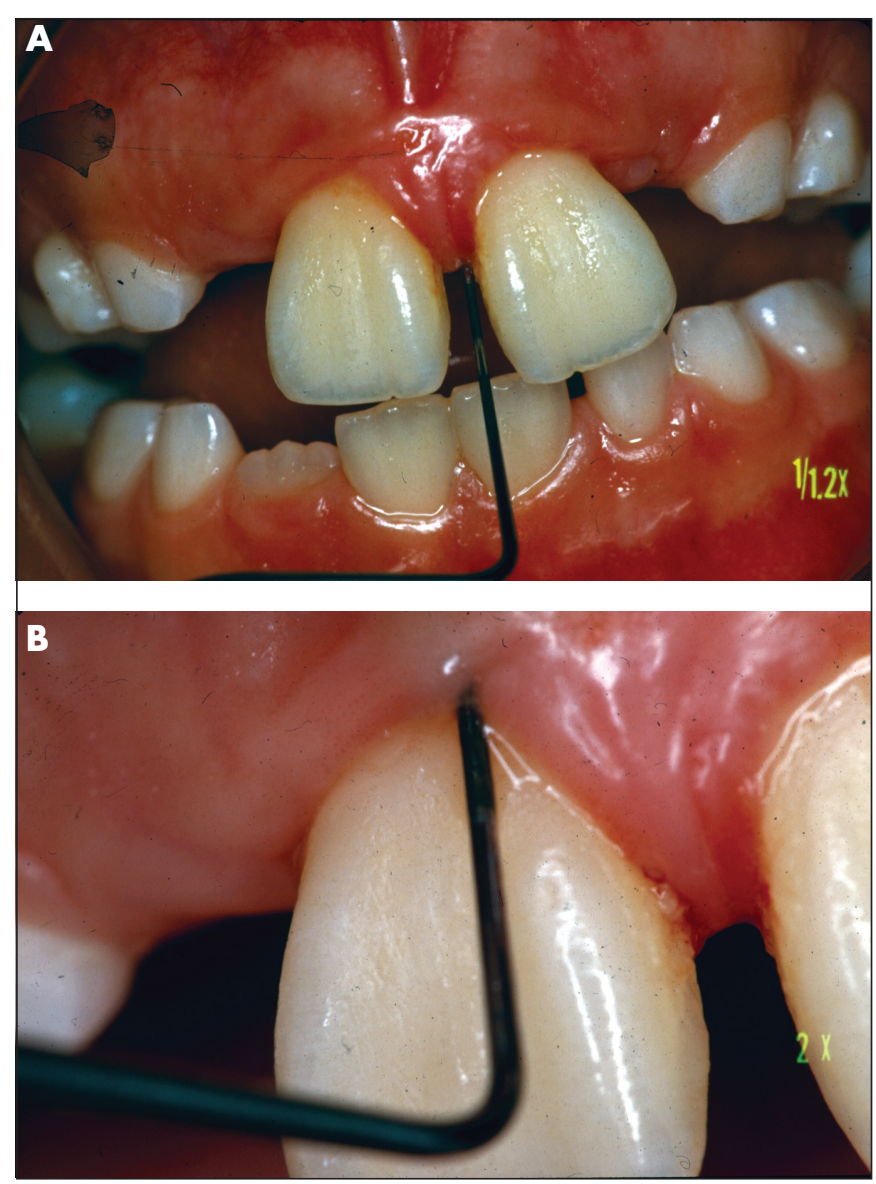

Fig. 1: Imagen clínica de un paciente de 8 años con periodontitis pre-puberal. Puede apreciarse la pérdida de inserción de $9 \mathrm{~mm}$ a nivel del 11 .

pueda ser atribuida a las poblaciones de estudio, así como a métodos diagnósticos y de clasificación de la enfermedad diferentes.

\section{Periodontitis refractaria}

Existe un grupo de pacientes, resistentes a un tratamiento periodontal adecuado, en los que la susceptibilidad genética parece tener un papel importante. Este grupo de pacientes con este patrón clínico de periodontitis, es el resultado de diferentes influencias que incluyen factores genéticos y exógenos que alteran la respuesta del huésped ante la agresión bacteriana (42). Algunos de estos factores son: el tabaco (43), diabetes, stress $(42,44)$ o aspectos microbiológicos (45). Aunque los resultado no son demasiado concluyentes en cuanto a una flora diferente de los pacientes refractarios respecto al resto de los pacientes (42), si 
son muchos los estudios que han encontrado alteraciones en el normal funcionamiento del sistema inmune $(43,46-49)$, alteraciones estas, con una base genética (42). (Figuras 2 A, B y C).

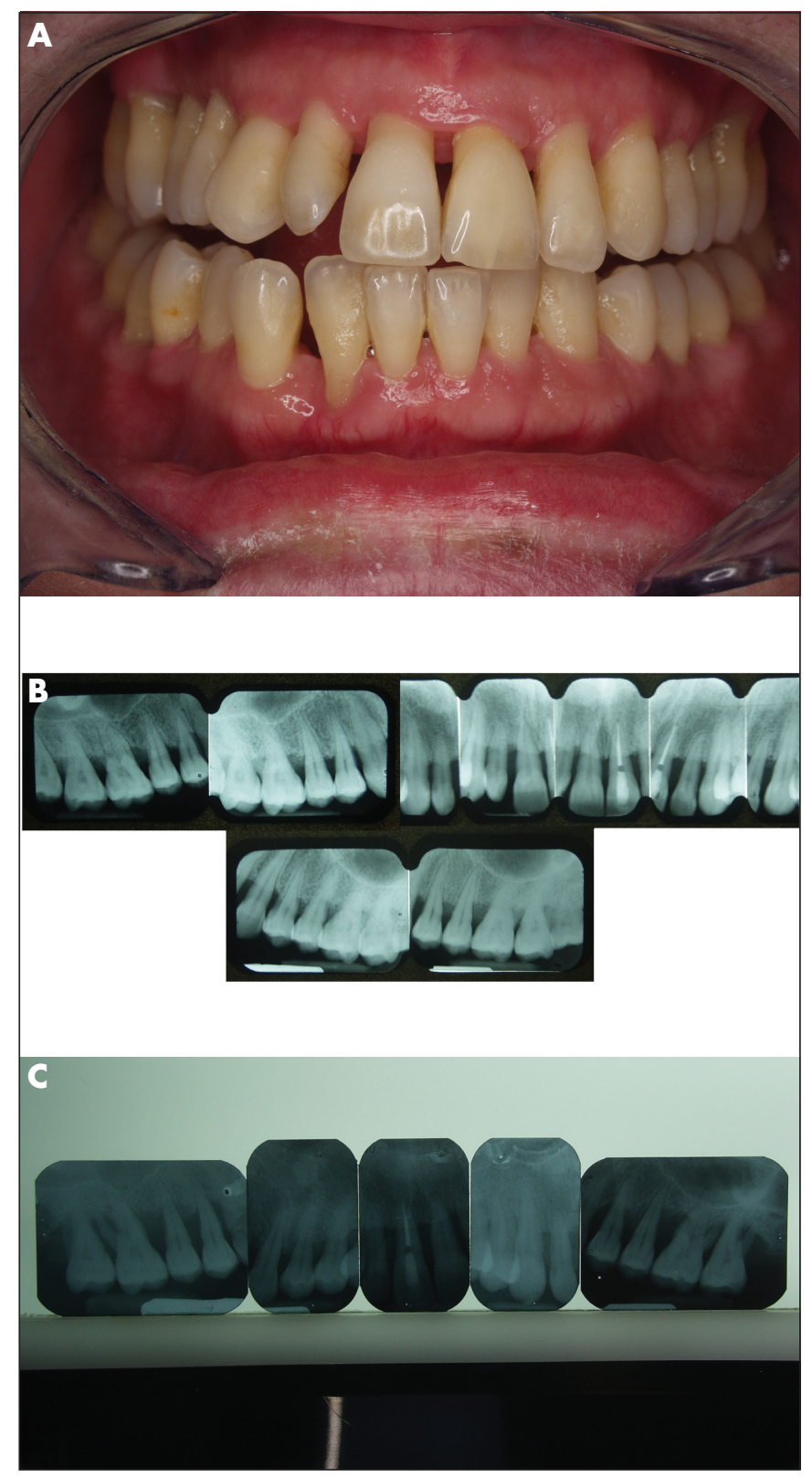

Fig. 2: A) Imagen clínica de una paciente con 37 años con una periodontitis refractaria. B) panorámica en el año 1999 donde se aprecia una pérdida de inserción del 50\%, la paciente recibió tratamiento básico y cirugía de los cuadrantes I, II y III. C) 7 años después, y a pesar del tratamiento periodontal y el mantenimiento, la pérdida de inserción ha progresado y se sitúa en la arcada superior en el $70 \%$.

\section{Desórdenes genéticos}

Otra línea de evidencia sobre la base genética para la aparición y desarrollo de la periodontitis, especialmente en las formas tempranas y severas de esta enfermedad es su asociación con desordenes genéticos hereditarios (1.2). Una característica común de estas condiciones es que son heredadas mediante un modelo simple mendeliano y que habitualmente se deben a alteraciones de un único gen. Esta alteración o mutación del gen y su correspondiente defecto bioquímico son normalmente conocidos. El estudio de estos desórdenes en los que la periodontitis es un hallazgo frecuente puede servirnos para desvelar el papel de determinados factores de riesgo genéticos para la enfermedad. Algunos de estos síndromes son:

- Síndrome de Chediak-Higashi: Esta enfermedad rara se transmite de forma autosómica recesiva. En estos pacientes hay una deficiencia en la competencia de los fagotitos debido a un defecto en el gen regulador de su actividad lisosómica (50) y son propensos a las infecciones en general. Algunos de estos pacientes padecen gingivitis severa, pérdida de hueso generalizada y pérdida prematura de los dientes (51).

- Neutropenia cíclica o permanente: Existen formas raras de agranulocitosis de carácter autonómico recesivo que se han asociado con periodontitis agresiva (52). El síndrome de Cohen cursa con neutropenia y se ha asociado con pérdida generalizada de hueso alveolar (53) Aunque las neutropenias se han asociado con mayor riesgo de padecer periodontitis, existen neutropenias benignas crónicas familiares con una expresión muy variable en las que no todos los individuos se ven afectados por infecciones recurrentes o periodontitis (54) (Figuras $3 \mathrm{~A}$ y B).

- Síndrome de Papillon-Lefevre (SPL): Se trata de un desorden autosómico recesivo caracterizado por hiperqueratosis palmoplantar y periodontitis de comienzo temprano (Figuras 4 A y B) (55). Pueden ser afectados ambos tipos de denticiones. Estudios de asociación con este síndrome han mostrado que está causado por la mutación del gen de la catepsina, localizado en el cromosoma 1 ( $(55,56)$. La catepsina $\mathrm{C}$ es una cistein-proteasa expresada de forma abundante en varios tejidos incluido el epitelio y algunas células del sistema inmune como los polimorfonucleares. Parece que funciona como una proteína de degradación y activación de proenzimas de las células inflamatorias e inmunes. 


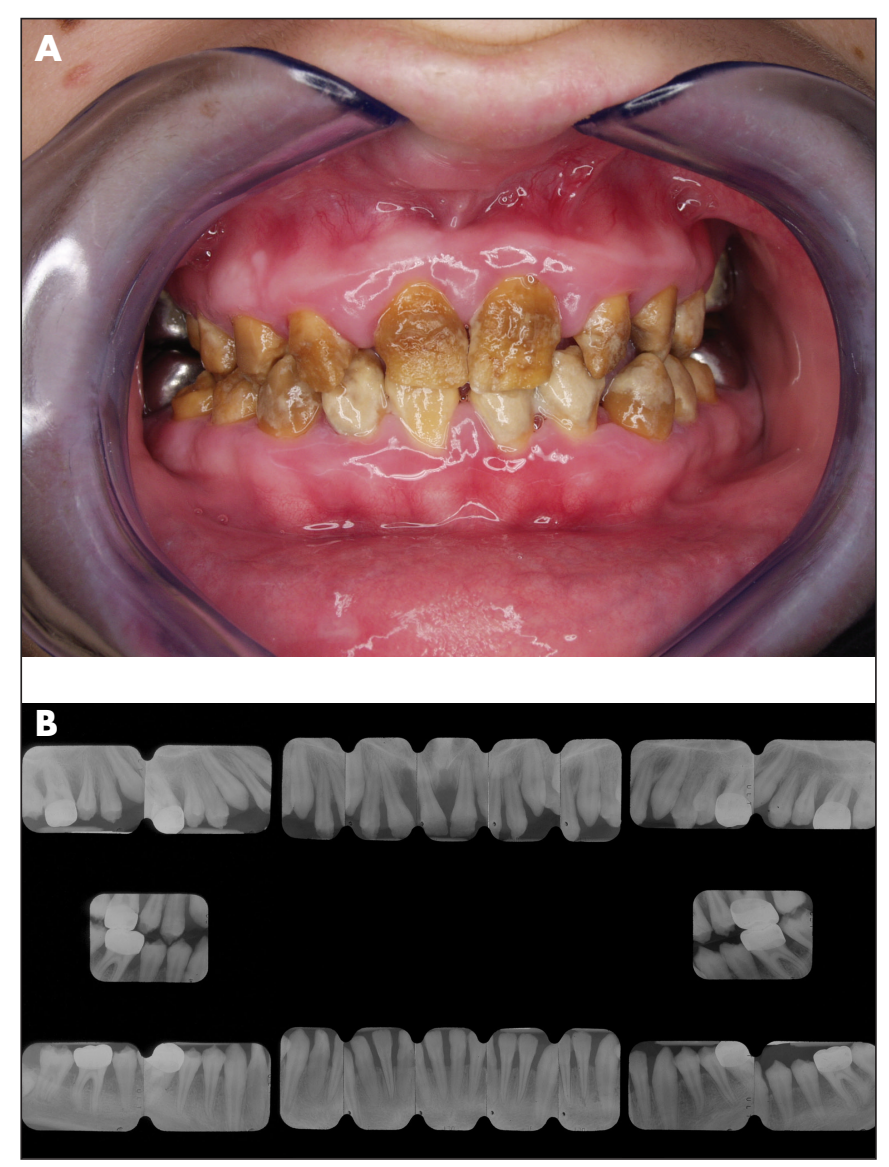

Fig. 3: Paciente de 12 años con neutropenia cíclica familiar y amolegénesis imperfecta $A$ ) imagen clínica. $B$ ) La pérdida de inserción es evidente en la radiografía.

A pesar de que el tratamiento periodontal convencional en este tipo de pacientes suele tener un pronóstico pobre, existen publicaciones recientes que han mostrado resultados satisfactorios a largo plazo con tratamientos basados en un diagnóstico precoz así como la utilización de una correcta terapia antibiótica guiada desde un punto de vista microbiológico $(56,57)$ En algunos paciente con SPL, la periodontitis de comienzo temprano está fuertemente asociada con el Actinobacillus actinomycetemcomitans y la destrucción periodontal puede detenerse si se elimina la bacteria (58).

- Hipofosfatasia: Es un desorden raro causado por mutaciones en el gen de la alkalin fosfatasa tisular no específica. La mutación resulta en una deficiencia de esta enzima que provoca una mineralización anormal del hueso, deformidades e hipoplasia del cemento. Existe una forma dominante y otra rece-
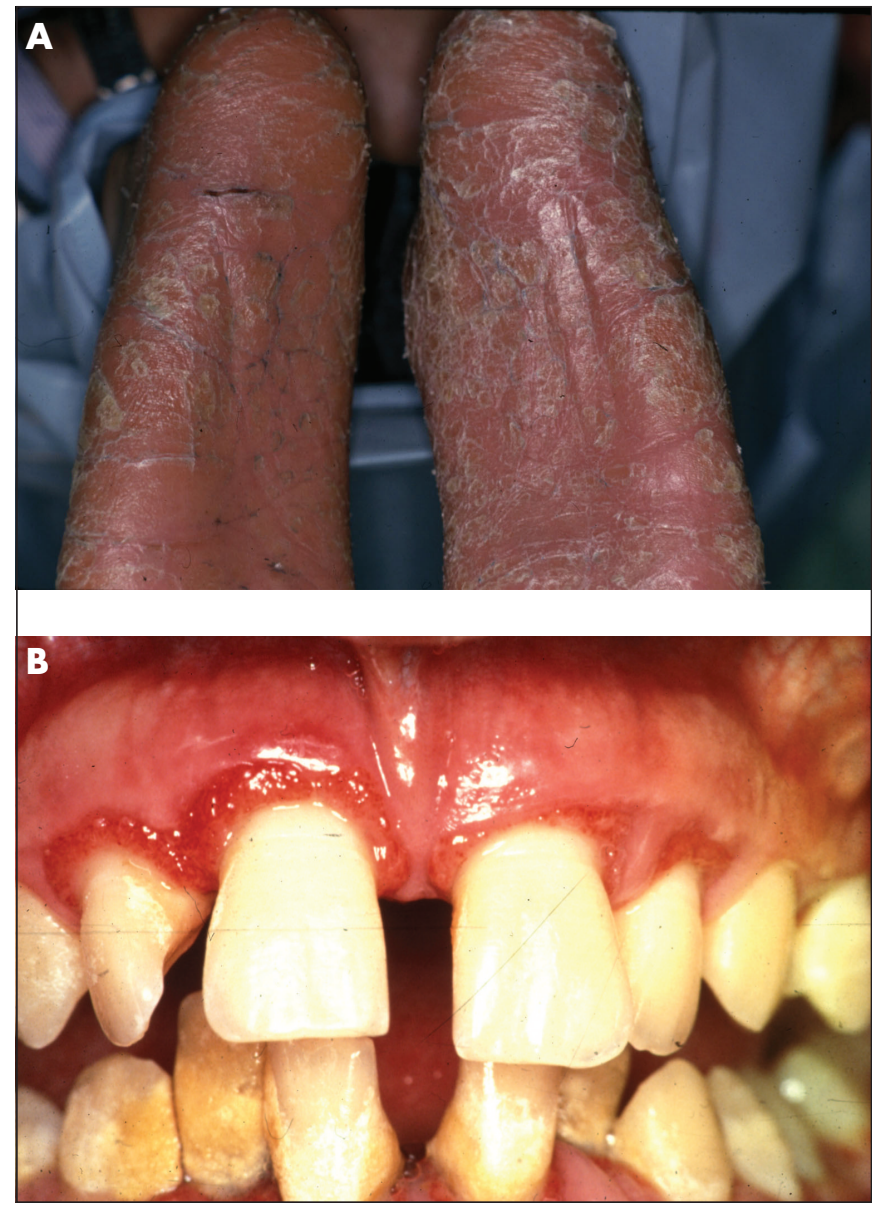

Fig. 4: A) Hiperqueratosis plantar típica de un paciente con síndrome de Papillon-Lefevre. B) Imagen clínica de la boca del mismo paciente. Se aprecia un colapso posterior de mordida y migración de los dientes anteriores como consecuencia de la pérdida de la dimensión vertical y pérdida de inserción.

siva. Esta condición resulta en una pérdida prematura de la dentición primaria y ocasionalmente de la secundaria (59).

- Síndrome de Ehler-Danlos: Este síndrome comprende un conjunto de desórdenes del tejido conectivo caracterizados por un defecto en la síntesis de colágeno. Ehler-Danlos tipo IV y VIII están asociados con un incremento en la susceptibilidad a la periodontitis (60) y se heredan de una forma autonómica dominante.

- Deficiencia de adhesión leucocitaria: Los pacientes con esta deficiencia tienen alto riesgo de desarrollar periodontitis de comienzo temprano, en especial periodontitis prepuberal. Esta deficiencia puede aparecer de dos formas, dependiendo del tipo 
de molécula de adhesión afectada (CD18 o CD15); ambas con rasgos autonómicos-recesivos. En ambos casos los leucocitos circulantes reducen sus receptores de superficie y no se adhieren a las células endoteliales vasculares, por tanto no son capaces de acumularse en las zonas de inflamación donde se requieren. Los individuos homocigotos que acarrean dos copias del alelo mutado experimentan una dramática reducción de la adhesión de los leucocitos, circunstancia que resulta en una propensión exagerada a las infecciones que pueden acarrear consecuencias fatales en la infancia. Los individuos que sobreviven, desarrollan periodontitis muy agresiva que suele comenzar con la erupción de los dientes deciduos (61).

La patogénesis de estas enfermedades, la gran cantidad de afectaciones sistémicas con que cursan, su asociación con otros posibles factores de riesgo en algunos casos y sobre todo la baja prevalencia de muchas de ellas hacen que la información que aporta su estudio en relación con la periodontitis se vea limitada acerca de su significación o extrapolación a la población general (27). No obstante, la importancia del análisis de estas condiciones o síndromes, es que demuestran claramente que una mutación genética puede aumentar de forma significativa la susceptibilidad a la periodontitis. Además, estas condiciones demuestran que la susceptibilidad genética puede segregarse mediante diferentes patrones de transmisión y puede potencialmente involucrar a diferentes genes.

\section{CONCLUSIONES}

En la aparición, desarrollo y severidad de la periodontitis intervienen factores microbiológicos, ambientales y genéticos. Aunque la placa bacteriana es el factor etiológico primario en la patogénesis de la periodontitis, su presencia por si sola, ya sea desde un punto de vista cuantitativo como cualitativo, no puede explicar la enorme variación de la enfermedad en la población. De las enfermedades periodontales, la predisposición genética parece ser más plausible para las periodontitis agresivas (de comienzo temprano) que en la periodontitis crónica (del adulto).

El estudio del patrón hereditario y algunos desórdenes genéticos simples en relación con la periodontitis pueden ayudarnos a comprender el papel de factores de riesgo genéticos para la enfermedad. La heterogenicidad de los estudios desde un punto de vista geográfico-étnico, así como las diferentes clasificaciones de enfermedad periodontal incluidas en las investigaciones dificultan la obtención de resultados concluyentes y extrapolables a la práctica clínica. Se necesitan por tanto más investigaciones en este emergente campo que permitan, no sólo conocer más sobre la etiología de la periodontitis, sino aplicar estos conocimientos en la práctica clínica diaria a la hora de establecer pronósticos y planes de tratamiento individualizados.

\section{ABSTRACT}

Periodontits is a multifactorial disease characterized for an inflammatory reaction which affects the teeth attachment apparatus. This inflammation is the consequence of the interaction of specific bacteria with the guest's immune response mechanisms. While the infection is a necessary requirement for the development of periodontitis, its progression and severity depends of a variety of environmental, behavioural, and genetic determinants. It has been proved that different subjects respond in a different way to the plaque formation. A limited but not underestimable fraction of the population is quite sensible to the disease progression at a relatively young age and a generalized loss of teeth takes place in a short time period. On the other hand, there is another part of the population who never develops this disease or in which the disease progression is slow and the loss of teeth during life is very small.

Although in the pathogenesis of the periodontits the bacterial plaque is the primary etiological factor, its presence alone can not answer the great variety of the disease development and progression. The aim of his study is to review the role that genetics plays in the different periodontal patterns by analyzing the scientific and clinical data that supports this question.

\section{KEY WORDS}

Periodontitis, genetic, polimorphism, risk factors.

\section{BIBLIOGRAFÍA}

1. Socransky SS, Haffjee AD. The bacterial etilogy of destructive periodontal disease. Current concepts. J Periodontol 1992; 63: 322-31. 
2. Alaluusua S, Asikainen S, Lai CH. Intrafamilial transmission of Actinobacillus actinomycetemcomitans. J Periodontol. 1991 Mar; 62(3): 207-10.

3. Offenbacher S. Periodontal disease: pathogenesis. Ann Periodontol 1996; 1: 821-78.

4. Norderyd O, Hugoson A, Grusovin G Risk of severe periodontal disease in a Swedish adult population. A longitudinal study. J Clin Periodontol. 1999 Sep; 26(9): 608-15.

5. Lindhe J, Ericsson I. Effect of ligature placement and dental plaque on periodontal tissue breakdown in the dog.J Periodontol. 1978 Jul;49(7):343-50.

6. Wiedemann W, Ketterle A, Naujoks R. [Dental studies on the protective effect of breast feeding] Dtsch Zahnarztl Z. 1979 May;34(5):427-9.

7. Van der Velden U, Abbas F, Armand S, Loos BG, Timmerman MF, Van der Weijden GA, Van Winkelhoff AJ, Winkel EG.Java project on periodontal diseases. The natural development of periodontitis: risk factors, risk predictors and risk determinants. J Clin Periodontol. 2006 Aug; 33(8): 540-8.

8. Loe H, Anerud A, Boysen H, Morrison E Natural history of periodontal disease in man. Rapid, moderate and no loss of attachment in Sri Lankan laborers 14 to 46 years of age. J Clin Periodontol. 1986 May; 13(5): 431-45.

9. Albandar JM Epidemiology and risk factors of periodontal diseases. Dent Clin North Am. 2005 Jul; 49 (3): 517-32.

10. Bergstrom J, Eliasson S, Dock J. A 10-year prospective study of tobacco smoking and periodontal health. J Periodontol. 2000 Aug; 71 (8): 1338-47.

11. Machuca G, Rosales I, Lacalle JR, Machuca C, Bullon P. Effect of cigarette smoking on periodontal status of healthy young adults. J Periodontol. 2000 Jan; 71 (1): 73-8.

12. Campus G, Salem A, Uzzau S, Baldoni E, Tonolo G. Diabetes and periodontal disease: a case-control study. J Periodontol. 2005 Mar;76(3):418-25.

13. Faria-Almeida R, Navarro A, Bascones A Clinical and metabolic changes after conventional treatment of type 2 diabetic patients with chronic periodontitis J Periodontol. 2006 Apr; 77(4): 591-8.
14. Kornman KS, Crane A, Wang HY, di Giovine FS, Newman MG, Pirk FW, Wilson TG Jr, Higginbottom FL, Duff GW. The interleukin-l genotype as a severity factor in adult periodontal disease. J Clin Periodontol. 1997 Jan; 24(1): 72-7.

15. Quinn SM, Zhang JB, Gunsolley JC, Schenkein HA, Tew JG. The influence of smoking and race on adult periodontitis and serum IgG2 levels. J Periodontol. 1998 Feb; 69(2):171-7.

16. Armitage GC. Periodontal diagnoses and classification of periodontal diseases. Periodontol 2000. 2004; 34: 9-21.

17. Beaty TH, Boughman JA, Yang P, Astemborski JA, Suzuki JB. Genetic analysis of juvenile periodontitis in families ascertained through an affected proband. Am J Hum Genet. 1987 May;40(5):443-52.

18. Van Dyke TE, Schweinebraten M, Cianciola LJ, Offenbacher S, Genco RJ Neutrophil chemotaxis in families with localized juvenile periodontitis. J Periodontal Res. 1985 Sep;20(5):503-14.

19. Marazita ML, Burmeister JA, Gunsolley JC, Koertge TE, Lake K, Schenkein HA. Evidence for autosomal dominant inheritance and race-specific heterogeneity in earlyonset periodontitis. J Periodontol. 1994 Jun;65(6):62330.

20. Stabholz A, Mann J, Agmon S, Soskolne WA. The description of a unique population with a very high prevalence of localized juvenile periodontitis. J Clin Periodontol. 1998 Nov;25(1 1 Pt 1):872-8.

21. Potter RH. Genetic studies of juvenile periodontitis. J Dent Res. 1990 Jan;69(1):94-5.

22. Boughman JA, Astemborski JA, Suzuki JB. Phenotypic assessment of early onset periodontitis in sibships.J Clin Periodontol. 1992 Apr; 19(4):233-9.

23. Asikainen S, Chen C, Alaluusua S, Slots J. Can one acquire periodontal bacteria and periodontitis from a family member? J Am Dent Assoc. 1997 Sep;128(9): 1263-71.

24. Boughman JA, Halloran SL, Roulston D, Schwartz S, Suzuki JB, Weitkamp LR, Wenk RE, Wooten R, Cohen MM. An autosomal-dominant form of juvenile periodontitis: its localization to chromosome 4 and linkage to dentinogenesis imperfecta and Gc. J Craniofac Genet Dev Biol. 1986;6(4):341-50. 
25. Hart TC, Marazita ML, McCanna KM, Schenkein HA, Diehl SR.Reevaluation of the chromosome $4 \mathrm{q}$ candidate region for early onset periodontitis. Hum Genet. 1993 Jun;91(5):416-22.

26. Michalowicz BS, Wolff LF, Klump D, Hinrichs JE, Aeppli DM, Bouchard TJ Jr, Pihlstrom BL.Periodontal bacteria in adult twins. J Periodontol. 1999 Mar;70(3):263-73.

27. Michalowicz BS. Genetic risk factors for the periodontal diseases. Compendium. 1994 Aug;15(8):1036, 1038, 1040 passim.

28. Michalowicz BS, Aeppli D, Virag JG, Klump DG, Hinrichs JE, Segal NL, Bouchard TJ Jr, Pihlstrom BL Periodontal findings in adult twins. J Periodontol. 1991 May;62(5): 293-9.

29. Michalowicz BS, Diehl SR, Gunsolley JC, Sparks BS, Brooks CN, Koertge TE, Califano JV, Burmeister JA, Schenkein HA. Evidence of a substantial genetic basis for risk of adult periodontitis.J Periodontol. 2000 Nov; 71(11):1699-707.

30. Moore WE, Moore LV. The bacteria of periodontal diseases. Periodontol 2000. 1994 Jun;5:66-77.

31. Amer A, Singh G, Darke C, Dolby AE. Association between HLA antigens and periodontal disease.Tissue Antigens. $1988 \mathrm{Feb}$;31(2):53-8.

32. Saxen L, Nevanlinna HR. Autosomal recessive inheritance of juvenile periodontitis: test of a hypothesis. Clin Genet. 1984 Apr;25(4):332-5.

33. Kinane DF, Shiba H, Hart TC. The genetic basis of periodontitis. Periodontol 2000. 2005; 39:91-117.

34. Kornman KS, Crane A, Wang HY, di Giovine FS, Newman MG, Pirk FW, Wilson TG Jr, Higginbottom FL, Duff GW. The interleukin-1 genotype as a severity factor in adult periodontal disease. J Clin Periodontol. 1997.

35. Salvi GE, Brown CE, Fujihashi K, Kiyono H, Smith FW, Beck JD, Offenbacher S. Inflammatory mediators of the terminal dentition in adult and early onset periodontitis. J Periodontal Res. 1998 May;33(4):212-25.

36. McDevitt MJ, Wang HY, Knobelman C, Newman MG, di Giovine FS, Timms J, Duff GW, Kornman KS. Interleukin1 genetic association with periodontitis in clinical practice. J Periodontol. 2000 Feb;71(2):156-63.
37. World Workshop in Clinical Periodontics (1989) American Academy of Periodontology, Chicago, III.

38. Altman LC, Page RC, Vandesteen GE, Dixon LI, Bradford C.Abnormalities of leukocyte chemotaxis in patients with various forms of periodontitis. J Periodontal Res. 1985 Nov;20(6):553-63.

39. Lopez NJ Clinical, laboratory, and immunological studies of a family with a high prevalence of generalized prepubertal and juvenile periodontitis. J Periodontol. 1992 May;63(5):457-68.

40. Shapira L, Schlesinger M, Bimstein E. Possible autosomal-dominant inheritance of prepubertal periodontitis in an extended kindred. J Clin Periodontol. 1997 Jun;24(6):388-93.

41. Boughman JA, Charon JA, Suzuki JB. Biological and genetical aspects of early onset periodontitis. J Parodontol. 1988 Sep;7(3):249-57.

42. Kornman KS.Refractory periodontitis: critical questions in clinical management. J Clin Periodontol. 1996 Mar;23(3 Pt 2):293-8.

43. MacFarlane GD, Herzberg MC, Wolff LF, Hardie NA. Refractory periodontitis associated with abnormal polymorphonuclear leukocyte phagocytosis and cigarette smoking. J Periodontol. 1992 Nov;63(11):908-13.

44. Haber J.Smoking is a major risk factor for periodontitis. Curr Opin Periodontol. 1994;:12-8.

45. Haffajee AD, Uzel NG, Arguello EI, Torresyap G, Guerrero DM, Socransky SS.Clinical and microbiological changes associated with the use of combined antimicrobial therapies to treat "refractory" periodontitis. J Clin Periodontol. 2004 Oct;31(10):869-77.

46. Handal T, Olsen I, Walker CB, Caugant DA. Betalactamase production and antimicrobial susceptibility of subgingival bacteria from refractory periodontitis. Oral Microbiol Immunol. 2004 Oct;19(5):303-8.

47. Kurdowska AK, Noble JM, Adcock JE.Interleukin-8 and anti-interleukin-8 autoantibodies in gingival crevicular fluid from patients with periodontitis. J Periodontal Res. $2003 \mathrm{Feb}$;38(1):73-8.

48. Oshrain HI, Telsey B, Mandel ID.Neutrophil chemotaxis in refractory cases of periodontitis.J Clin Periodontol. 1987 Jan; 14(1):52-5. 
49. Diagnostic potential of host response mediators. Adv Dent Res 1993 Aug;7(2):175-81.

50. Meyle J, Gonzales JR.Influences of systemic diseases on periodontitis in children and adolescents. Periodontol 2000. 2001;26:92-112.

51. Shibutani T, Gen K, Shibata M, Horiguchi Y, Kondo N, Iwayama Y.Long-term follow-up of periodontitis in a patient with Chediak-Higashi syndrome. A case report. J Periodontol. 2000 Jun;71(6):1024-8.

52. Saglam F, Atamer T, Onan U, Soydinc M, Kirac K.Infantile genetic agranulocytosis (Kostmann type). A case report. J Periodontol. 1995 Sep;66(9):808-10.

53. Alaluusua S, Kivitie-Kallio S, Wolf J, Haavio ML, Asikainen $\mathrm{S}$, Pirinen S.Periodontal findings in Cohen syndrome with chronic neutropenia. J Periodontol. 1997 May;68 (5):473-8.

54. Deasy MJ, Vogel RI, Macedo-Sobrinho B, Gertzman G, Simon B.Familial benign chronic neutropenia associated with periodontal disease. A case report. J Periodontol. 1980 Apr;51(4):206-10.

55. Ahuja V, Shin RH, Mudgil A, Nanda V, Schoor R. PapillonLefevre syndrome: a successful outcome. J Periodontol. 2005 Nov;76(11):1996-2001.

56. Pacheco JJ, Coelho C, Salazar F, Contreras A, Slots J, Velazco CH.Treatment of Papillon-Lefevre syndrome periodontitis. J Clin Periodontol. 2002 Apr;29(4): 370-4.
57. Lundgren T, Renvert S.Periodontal treatment of patients with Papillon-Lefevre syndrome: a 3-year follow-up. J Clin Periodontol. 2004 Nov;31(11):933-8.

58. Kamma JJ, Nakou M, Gmur R, Baehni PC.Microbiological profile of early onset/aggressive periodontitis patients. Oral Microbiol Immunol. 2004 Oct;19(5):314-21.

59. Baab DA, Page RC, Ebersole JL, Williams BL, Scott CR. Laboratory studies of a family manifesting premature exfoliation of deciduous teeth. J Clin Periodontol. 1986 Aug;13(7):677-83.

60. Hart TC, Kornman KS Genetic factors in the pathogenesis of periodontitis. Periodontol 2000. 1997 Jun; 14: 202-15.

61. Waldrop TC, Anderson DC, Hallmon WW, Schmalstieg FC, Jacobs RL. Periodontal manifestations of the heritable Mac-1, LFA-1, deficiency syndrome. Clinical, histopathologic and molecular characteristics.J Periodontol 1987 Jun;58(6):400-16.

62. Denny RE. Heredity and its influence on teeth. Dental Cosmos. 1930; 72:596-605.

\section{CORRESPONDENCIA}
Daniel Rodrigo Gómez
Clínica DASER Periodoncia e Implantes
C/ Alvarfañez de Minaya, 2
Guadalajara
e-mail:dperio@hotmail.com 